\title{
Artigo/Article
}

\section{Avaliação oftalmológica em pacientes com AIDS e neurotoxoplasmose}

\author{
Ophthalmological evaluation of patients with AIDS and neurotoxoplasmosis
}

Jane Mary Alves ${ }^{1,2}$, Vera Magalhães ${ }^{3}$ e Marcus Augusto Gomes de Matos $^{4}$

\begin{abstract}
RESUMO
Introdução: Descrever os achados fundoscópicos em pacientes com AIDS e neurotoxoplasmose em fase ativa. Métodos: Foi desenvolvido estudo prospectivo tipo série de casos incluindo 70 pacientes, de ambos os sexos, com idade variando de 20 a 63 anos, internados nas enfermarias de três hospitais públicos da Cidade do Recife, Pernambuco, com diagnósticos de AIDS e neurotoxoplasmose firmados segundo os critérios do Centers for Disease Control and Prevention (1992), no período de janeiro a outubro de 2008. Os pacientes se caracterizavam por: primeiro episódio de neurotoxoplasmose (65; 92,9\%) ou recidiva ( $5 ; 7,1 \%)$; desconhecimento de ter AIDS (23; 32,9\%), contagem média de linfócitos T CD4 de $139,8 \pm 3,04$ células $/ \mathrm{mm}^{3}$ e carga viral média igual a $137.080 \pm 39.380$ cópias $/ \mathrm{mL}$. Todos foram submetidos a exame oftalmológico, consistindo de: inspeção ocular; aferição da acuidade visual; investigação da função muscular extrínseca ocular e fundoscopia, empregando oftalmoscópio indireto binocular (modelo OHN3.5 (Eyetec) e ente externa de 20 dioptrias (Volk ${ }^{\circ}$. Resultados: Os achados consistiram em: exsudatos algodonosos retinianos $(8,6 \%)$, constricção arteriolar difusa leve (8,6\%); lesões de retinocoroidite cicatricial, características de toxoplasmose ocular $(5,7 \%)$, atrofia do epitélio pigmentar retiniano (2,9\%), descolamento da retina $(2,9 \%)$, aumento de escavação papilar $(1,4 \%)$, degeneração periférica retiniana $(1,4 \%)$, macroaneurisma $(1,4 \%)$, papiledema bilateral $(1,4 \%)$, tração vítreo-retiniana $(1,4 \%)$. Conclusões: Pacientes com AIDS e neurotoxoplasmose podem apresentar alterações fundoscópicas características da toxoplasmose ocular, na forma ativa ou cicatricial, relacionadas ao HIV ou, ainda, a outras doenças oportunistas ou sistêmicas, podendo ser de grande auxílio num tratamento integral do paciente por uma equipe multiprofissional.
\end{abstract}

Palavras-chaves: Toxoplasmose ocular. AIDS. Infecções oculares. Fundo de olho.

\begin{abstract}
Introduction: To describe fundoscopic findings among patients with AIDS and active-phase neurotoxoplasmosis. Methods: A prospective study of case series type was developed, including 70 patients of both sexes and ages ranging from 20 to 63 years who were admitted to the wards of three public hospitals in the City of Recife, Pernambuco, Brazil, from January to October 2008, with diagnoses of AIDS and neurotoxoplasmosis determined according to the criteria of the Centers for Disease Control and Prevention (1992). The patients were characterized by a first episode of neurotoxoplasmosis $(65 ; 92.9 \%)$ or recurrence $(5 ; 7.1 \%)$, unawareness of having AIDS $(23 ; 32.9 \%)$, mean T CD 4+ count of $139.8 \pm 3.04$ lymphocytes $/ \mathrm{mm}^{3}$ and mean viral load of $137,080 \pm 39,380$ copies $/ \mathrm{ml}$. All patients underwent ophthalmological examination consisting of ocular inspection, gauging of visual acuity, investigation of ocular extrinsic muscle function and fundoscopy using a binocular indirect ophthalmoscope (model OHM 3.5 Eyetec $^{\circ}$ ) and external lens of 20 diopters (Volk ${ }^{\circ}$. Results: The findings consisted of retinal cotton-wool spot exudates $(8.6 \%)$, slight diffuse arteriolar constriction (8.6\%), retinochoroiditis scars characteristic of ocular toxoplasmosis (5.7\%), atrophy of retinal pigment epithelium (2.9\%), retinal detachment (2.9\%), increased papillary excavation $(1.4 \%)$, retinal peripheral degeneration $(1,4 \%)$, macroaneurysm $(1.4 \%)$, bilateral papilledema (1.4\%) and vitreous-retinal traction (1.4\%). Conclusions: Patients with AIDS and neurotoxoplasmosis may present fundoscopic abnormalities characteristic of ocular toxoplasmosis, either in active or in scar form, related to HIV or even to other opportunist or systemic diseases, which can be of great aid for integral treatment of patients by a multiprofessional team.
\end{abstract}

Key-words: Ocular toxoplasmosis. AIDS. Ocular infections. Fundoscopy.

1. Pós-Graduação, Universidade Federal de Pernambuco, Recife, PE. 2. Instituto de Olhos de Recife, Recife, PE. 3. Departamento de Doenças Infecciosas e Parasitárias, Universidade Federal de Pernambuco, Recife, PE. 4. Real Visão, Hospital Português, Recife, PE.

Endereço para correspondência: Dra . Jane Mary Alves. Rua Dom Sebastião Leme 57/202, Graças, 52011-160 Recife, PE.

Tel: $55813423-9298$

e-mail: janealves.alves@bol.com.br

Recebido para publicação em 17/08/2009

Aceito em 08/01/2010

\section{INTRODUÇÃO}

Desde a descrição dos primeiros casos de AIDS, a importância do envolvimento ocular é evidente $^{1}$ e muito importante, porque cerca de $60 \%$ dos pacientes desenvolvem tais manifestações em alguma fase do curso da AIDS $^{2}$.

À fundoscopia, pacientes com AIDS podem apresentar alterações da microcirculação ocular (exsudatos algodonosos, microaneurismas e microhemorragias), infecções oportunistas secundárias (retinopatia por Citomegalovírus, por toxoplasmose, herpética, sifilítica, coroidite pelo Cryptococcus neoformans e pelo Pneumocistis jirovesi), e distúrbios neuroftalmológicos, dentre os quais estão o papiledema ${ }^{3}$.

Nos casos em que as manifestações oculares na AIDS associam-se à infecção oportunista secundária por toxoplasmose, o acometimento ocular é ainda mais importante porque reduz a qualidade de vida dos pacientes, devido à significativa diminuição da acuidade visual ${ }^{4,5}$. Além disso, nesses casos as lesões podem ter aparência clínica atípica, seguir um curso incomum e demonstrarem refratariedade ao tratamento convencional ${ }^{6}$. Nesses pacientes, o acometimento ocular ocorre com uma frequência que varia de $21,4 \%$ a $95 \%$, sendo menor em pacientes não hospitalizados ${ }^{7-9}$.

No entanto, no segmento posterior do olho de pacientes com AIDS as alterações causadas pelo Toxoplasma gondii tem frequência menor e variável em função da prevalência do parasita na população ${ }^{7}$. Nos países em desenvolvimento, podem estar presentes em $2 \%$ a $10 \%$ dos pacientes com $\operatorname{AIDS}^{10}$. No Brasil, foram relatadas frequências de $16,7 \%{ }^{11}$, em Santa Catarina e de $21 \%$ na população geral de Erechin, Rio Grande do Sul ${ }^{7}$.

É importante ressaltar que pacientes com AIDS e acometimento do segmento posterior do olho por toxoplasmose podem ser pouco sintomáticos na fase inicial da doença ou mesmo não apresentar queixas oculares. Este fato associado às manifestações atípicas pode comprometer o tratamento precoce e o acompanhamento adequado, que poderiam evitar a evolução do quadro para complicações graves, como 
catarata, descolamento de retina e glaucoma ${ }^{12}$. Daí a importância do conhecimento das lesões fundoscópicas típicas ou prováveis.

O objetivo deste artigo foi descrever os achados fundoscópicos em pacientes com AIDS e neurotoxoplasmose em fase ativa, para compreender melhor a doença na sua totalidade e melhorar a qualidade de vida do paciente, com intervenções adequadas a cada caso.

\section{MÉTODOS}

No período de janeiro a outubro de 2008, foi desenvolvido estudo tipo série de casos, envolvendo 70 pacientes, com idade igual ou maior que 20 anos, internados nas enfermarias de três hospitais públicos da cidade do Recife, Pernambuco (Hospital Correa Picanço, Hospital das Clínicas da Universidade Federal de Pernambuco e Hospital Universitário Oswaldo Cruz da Universidade de Pernambuco), soro positivos para HIV e toxoplasmose (IgG), com diagnósticos de AIDS e de acometimento do sistema nervoso central pelo Toxoplasma gondii. Foram excluídos os pacientes com história de alergia a colírio midriático ou presença de câmara ocular anterior rasa, pelo risco de crise de glaucoma agudo.

A caracterização dos pacientes foi obtida por meio de consulta ao prontuário e compreendeu: dados sociodemográficos, carga viral, contagem de linfócitos T CD4 em sangue periférico e presença de comorbidades sistêmicas.

O diagnóstico da neurotoxoplasmose foi realizado por infectologista ou por neurologista, obedecendo aos critérios do Center for Disease Control and Prevention ${ }^{13}$.

Clinicamente, baseou-se na presença de cefaléia, alterações de status mental, febre, sinais neurológicos focais, como fraqueza muscular, distúrbios de fala, tontura, alterações em pares cranianos e de campo visual, distúrbios sensitivos, disfunção cerebelar, meningismo, incoordenação motora e manifestações neuropsiquiátricas. Baseou-se também nos achados típicos de imagem por ressonância magnética ou tomografia computadorizada e na melhora clínica com o tratamento instituído.

Todos os pacientes foram submetidos ao exame oftalmológico, o qual consistiu de: inspeção ocular com lanterna, dirigindo o feixe luminoso paralelamente ao ponto de junção palpebral externo, para avaliação da profundidade da câmara anterior; aferição da acuidade visual para perto, de cada olho, utilizando os optotipos de Jaeger colocados a $33 \mathrm{~cm}$ do globo ocular e investigação da função muscular extrínseca ocular. A fundoscopia foi realizada por um retinólogo uveólogo juntamente com a pesquisadora, com oftalmoscópio indireto binocular (OBI), com iluminação elétrica halógena, modelo OHN 3.5 (Eyetec ${ }^{\circ}$ ), e lente externa de 20 dioptrias (Volk), após instilação em cada olho de 1 gota de colírio de tropicamida a $1 \%$ no saco conjuntival de cada olho, a cada 10 minutos, por três vezes, para promover midríase.

Os pacientes com diagnóstico de alterações fundoscópicas foram submetidos a: retinografia, empregando retinógrafo digital (Topcon", Japão), avaliada pelo programa Image NET, para processamento das imagens, assim como angiofluoresceinografia, quando indicada, após infusão endovenosa periférica rápida de $5 \mathrm{~mL}$ de fluoresceína sódica a $10 \%$.

Foram excluídos os diagnósticos oculares diferenciais de infecções oportunistas secundárias por Citomegalovírus, sifilítica e herpética.
Os pacientes que necessitaram de tratamento oftalmológico foram acompanhados pela pesquisadora e pelo retinólogo uveólogo.

A retinocoroidite foi classificada segundo atividade, tipicidade e topografia. Quanto à atividade, a retinocoroidite foi considerada: cicatricial, na presença de margens bem delimitadas, graus variados de hiperplasia do epitélio pigmentário e atrofia retinocoroidea; ou ativa, quando foi possível observar lesão branco-amarelada, ocasionalmente cinza, com limites mal definidos devido ao edema retiniano circunjacente. Quanto à tipicidade, a lesão foi classificada como: típica quando cicatrizada ou ativa, satélite a uma ou mais lesões cicatrizadas, ou provável, quando necrosante, focal típica, porém isolada, podendo estar em atividade ou cicatrizada. Quanto à topografia, as lesões foram classificadas como periféricas ou em pólo posterior.

Os dados foram organizados e analisados com o programa Excel. As variáveis nominais e ordinais foram expressas por distribuição de frequência absoluta e relativa. A variável idade foi resumida empregando-se os parâmetros de média e erro padrão da média, da estatística descritiva.

\section{Ética}

A pesquisa foi aprovada pelo Comitê de Ética da Universidade Federal de Pernambuco e registrada sob o número CEP/CCS/ UFPE no $371 / 07$.

\section{RESULTADOS}

Dentre os 70 pacientes com toxoplasmose do sistema nervoso central, houve predomínio do gênero masculino (72,9\%). A idade variou de 20 a 63 anos com média de 34,3 $\pm 1,1$ anos, sendo maior entre os pacientes do gênero masculino $(34,9 \pm 1,2$ anos) do que no feminino ( $32,6 \pm 2,2$ anos). Foram mais frequentes pacientes solteiros $(62,9 \%)$ e procedentes da região metropolitana do Recife $(82,9 \%)$, como mostra a Tabela 1.

Constatou-se que $23(32,9 \%)$ dos pacientes declararam desconhecer o fato de ser portador do HIV e não associaram seus sintomas à AIDS.

Na Tabela 2, as comorbidades sistêmicas mais frequentes foram candidíase $(30 \%)$, tuberculose $(17,1 \%)$, diarréia $(11,4 \%)$, hipertensão arterial $(10 \%)$ e escabiose $(8,6)$. Três $(4,3 \%)$ pacientes eram esquizofrênicos.

Na Tabela 3, estão expressos os achados fundoscópicos, na qual se observa maior frequência de exsudatos algodonosos retinianos (Figura 1A) e constrição arteriolar difusa leve (Figura 1B), ambos acometendo $6(8,6 \%)$ pacientes.

Quatro $(5,7 \%)$ pacientes apresentavam lesões de retinocoroidite cicatricial, características de toxoplasmose ocular. Em um (25\%) paciente, a lesão era periférica, do tipo provável (Figura 1C); em um (25\%), a lesão em olho direito era cicatricial típica, de localização periférica (Figura 1D), e em outro caso, a lesão em olho esquerdo era típica, localizada em pólo posterior (Figura 1E). Um paciente apresentava em olho direito lesões típicas, múltiplas, uma delas acometendo mácula, contração vítreo-retiniana, resultando em importante baixa da acuidade visual, além de lesão cicatricial, provável, periférica em olho esquerdo (Figuras 1F, 1G). Os demais achados fundoscópicos estão apresentados na Figura 2.

Não se diagnosticou qualquer caso de retinocoroidite ativa, fosse ela típica ou provável. 
TABELA 1 - Distribuição das variáveis sociodemográficas de 70 pacientes com AIDS e neurotoxoplasmose - Recife, janeiro/outubro 2008.

\begin{tabular}{lcc}
\hline Variáveis sociodemográficas & Frequência & Percentual \\
\hline Gênero & 51 & 72,9 \\
masculino & 19 & 27,1 \\
feminino & & \\
\hline Idade (anos) & 25 & 35,7 \\
$20-29$ & 32 & 45,7 \\
$30-39$ & 13 & 18,6 \\
\hline 40 & & \\
\hline Estado civil & 44 & 62,9 \\
solteiro & 22 & 31,4 \\
casado & 3 & 4,3 \\
viúvo & 1 & 1,4 \\
\hline divorciado & & 82,9 \\
\hline Procedência & 58 & 17,1 \\
\hline Região Metropolitana do Recife & 12 & \\
outros municípios & & \\
\hline
\end{tabular}

TABELA 2 - Distribuição das comorbidades sistêmicas de 70 pacientes com AIDS e neurotoxoplasmose - Recife, janeiro/outubro 2008.

\begin{tabular}{|c|c|c|}
\hline Comorbidades sistêmicas & Frequência & Percentual $^{*}$ \\
\hline Candidíase & 21 & 30,0 \\
\hline Tuberculose & 12 & 17,1 \\
\hline Diarréia & 8 & 11,4 \\
\hline Hipertensão arterial & 7 & 10,0 \\
\hline Escabiose & 6 & 8,6 \\
\hline Herpes & 5 & 7,1 \\
\hline Infecção do trato urinário & 3 & 4,3 \\
\hline Esquizofrenia & 3 & 4,3 \\
\hline Diabetes mellitus & 1 & 1,7 \\
\hline Anemia & 1 & 1,4 \\
\hline Condiloma gigante anal & 1 & 1,4 \\
\hline Miocardiopatia & 1 & 1,7 \\
\hline Ascite & 1 & 1,7 \\
\hline Dermatite seborréica & 1 & 1,7 \\
\hline Edema em membros inferiores & 1 & 1,4 \\
\hline Litíase renal & 1 & 1,4 \\
\hline Otite média externa & 1 & 1,4 \\
\hline Desnutrição & 1 & 1,4 \\
\hline Hemorróidas & 1 & 1,4 \\
\hline Tinea cruris & 1 & 1,4 \\
\hline Gastrite com Helicobacter pylori positivo & 1 & 1,4 \\
\hline
\end{tabular}

*percentuais calculados com base num total de 70 pacientes.
TABELA 3 - Distribuição dos achados fundoscópicos de 70 pacientes com AIDS e neurotoxoplasmose - Recife, janeiro/outubro 2008.

\begin{tabular}{lcr}
\hline Achados fundoscópicos & Frequência & Percentual $^{*}$ \\
\hline Exsudatos algodonosos retinianos & 6 & 8,6 \\
Constrição arteriolar difusa leve & 6 & 8,6 \\
Retinocoroidite cicatricial típica & 3 & 4,3 \\
Atrofia do epitélio pigmentar da retina & 2 & 2,9 \\
Descolamento de retina & 2 & 2,9 \\
Aumento da escavação papilar & 1 & 1,4 \\
Degeneração retiniana periférica (lattice) & 1 & 1,4 \\
Macroaneurismas & 1 & 1,4 \\
Papiledema bilateral & 1 & 1,4 \\
Tração vítreo-retiniana & 1 & 1,4 \\
Retinocoroidite cicatricial provável & 1 & 1,4 \\
\hline
\end{tabular}

*percentuais calculados com base num total de 70 pacientes.
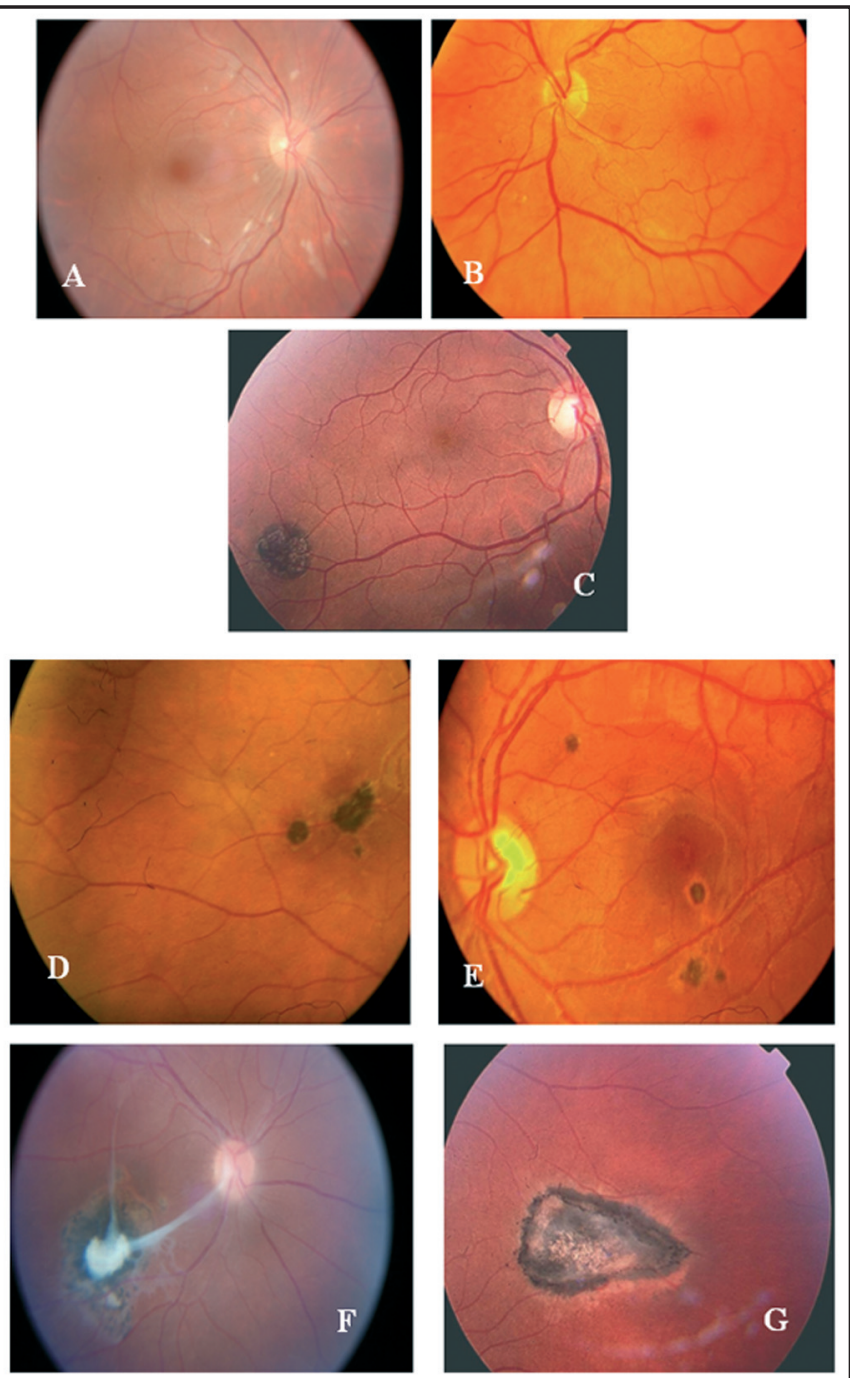

Fonte: Fotos da Pesquisadora

FIGURA 1 - Achados fundoscópicos. A) Exsudatos retinianos algodonosos em olho direito em paciente com AIDS e neurotoxoplasmose. B) Constrição arteriolar difusa leve em olho esquerdo em paciente com AIDS e neurotoxoplasmose. C) Retinocoroidite periférica cicatricial provável em olho em paciente com AIDS e neurotoxoplasmose. D) Retinocoroidite cicatricial típica periférica em olho direito. E) Retinocoroidite cicatricial típica em polo posterior em olho esquerdo. F) Retinocoroidite macular, típica, com tração vítreo-retiniana (sinal de Franceschetti) em olho direito. G) Retinocoroidite cicatricial provável periférica em olho esquerdo. 


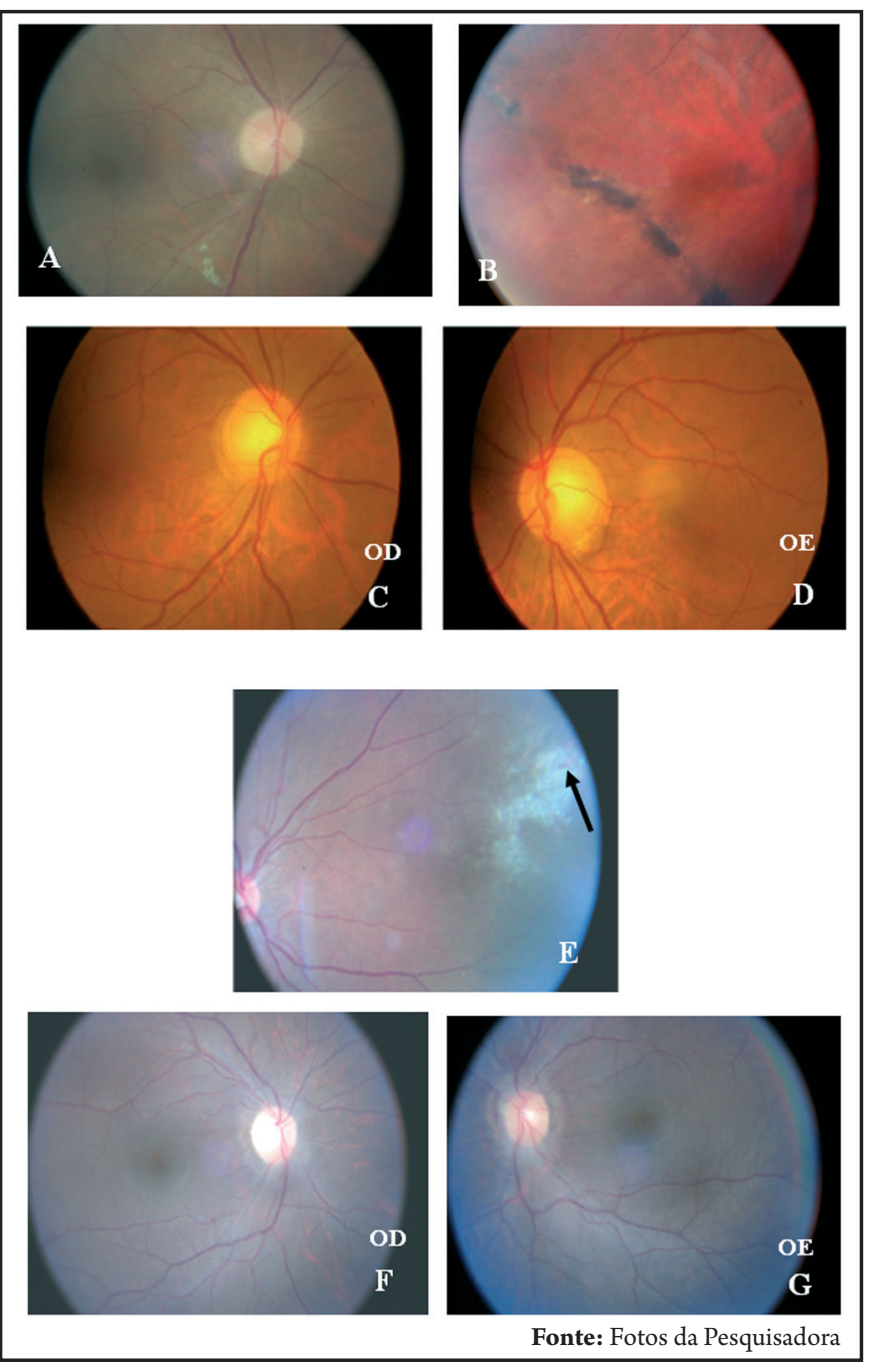

FIGURA 2 - Achados fundoscópicos. A) Atrofia do epitélio pigmentar da retina constrição arteriolar e exsudatos retinianos em olho direito em paciente com AIDS e neurotoxoplasmose. B) Lesão degenerativa retiniana periférica (lattice) em paciente com AIDS e neurotoxoplasmose. C, D) Aumento bilateral da escavação papilar em paciente com AIDS e neurotoxoplasmose. E) Macroaneurisma arterial e edema retiniano em olho esquerdo. Observar que o macroaneurisma arterial está circundado por depósitos esbranquiçados sugestivos de exsudatos, em cujo interior identifica-se um foco hemorrágico retiniano (seta). F, G) Papiledema bilateral em paciente com AIDS e neurotoxoplasmose.

\section{DISCUSSÃO}

Classificando em categorias as lesões oculares, que acometem pacientes com $\mathrm{AIDS}^{14}$, constituíram-se manifestações decorrentes de alteração da microvasculatura os exsudatos algodonosos retinianos, o macroaneurisma e a constrição arteriolar; as alterações oculares por infecções oportunistas secundárias à AIDS incluíram a retinocoroidite cicatricial, característica da toxoplasmose e o descolamento de retina, atribuído à uveíte posterior, decorrente de infecção por Citomegalovírus. Como alteração neuroftalmológica foi diagnosticado o papiledema. Em nenhum paciente havia presença de tumor.

O predomínio de exsudatos algodonosos retinianos, da presente pesquisa, corroborou os achados de outros autores ${ }^{5,12,15}$. No entanto, a frequência de $8,6 \%$ foi menor que a estimativa de $40 \%$ a $100 \%$. Essa diferença deve ser analisada com precaução, porque a estimativa se refere à presença desses exsudatos em alguma fase da vida do paciente com AIDS, característica que esteve ausente na amostra analisada já que 32,9\% dos casos foram examinados à primeira manifestação clínica da AIDS. Além disso, os exsudatos algodonosos são efêmeros ${ }^{15}$.

A frequência de exsudatos algodonosos retinianos foi maior que os $7 \%$ referidos em um estudo envolvendo 445 pacientes, com soropositividade para HIV, dos quais $22 \%$ apresentavam neurotoxoplasmose ${ }^{16}$. No entanto, foi menor que os $10 \%$ relatados na pesquisa com 1.100 pacientes HIV positivos, atendidos em regime ambulatorial ${ }^{15}$ e os $13 \%$, diagnosticados em 77 pacientes com AIDS ${ }^{12}$.

A frequência do presente estudo pode ter como causa um grau de imunodeficiência mais acentuado e a concomitância com neurotoxoplasmose em todos os casos, raramente referida em trabalhos que abordam achados oftalmológicos na AIDS. Os autores não restringem a amostra, como no presente estudo, a pacientes com AIDS e neurotoxoplasmose $\mathrm{e}^{5,12}$. Um único trabalho retrospectivo, incluindo pesquisa a 142 prontuários, adotou essa metodologia e referiu taxa de $6,7 \%$, mas não detalhou a época do curso da neurotoxoplasmose em que o exame fundoscópico foi realizado ${ }^{17}$.

$\mathrm{O}$ achado macroaneurisma associado a edema e hemorragia retiniana em um paciente, não integra as manifestações oculares daAIDS ou da neurotoxoplasmose, e pode ter decorrido de hiperviscosidade sanguínea ou de oclusão do ramo da veia central da retina. Como a investigação de hiperviscosidade sanguínea foi negativa nesse paciente, admitiu-se a possibilidade de oclusão venosa retiniana.

A constrição arteriolar difusa leve é uma alteração da microvasculatura retiniana, mas não pôde ser atribuída à AIDS nesses casos, porque havia concomitância com hipertensão arterial, embora as alterações fundoscópicas da AIDS sejam polimorfas ${ }^{18}$. Esse achado pareceu indicar a importância de o oftalmologista investigar, na anamnese, a presença de comorbidades, para afastar uma associação espúria entre achados fundoscópicos e AIDS.

Coincidiram com a literatura os achados de lesões fundoscópicas secundárias a doenças oportunistas em pacientes com AIDS, importantes porque exercem maior impacto na função visual. Estiveram presentes as retinocoroidites provável e típica, com tração vítreo-retiniana (sinal de Franceschetti), na forma cicatricial, de origem toxoplásmica (5,7\%) e o descolamento de retina, atribuído à infecção por citomegalovírus $(2,9 \%)$ estiveram presentes. Talvez pela incidência de toxoplasmose no Brasil ser alta, nossos achados contrariaram trabalhos de outros países que relatam porcentagem de $1 \%$ de toxoplasmose ocular e maior, de lesões secundárias ao citomegalovírus $^{19}$.

Dentre as lesões neuroftalmológicas, o papiledema bilateral foi diagnosticado em um paciente, correspondendo a um percentual menor do que o referido por Zajdenweber $\operatorname{cols}^{17}$. O achado, em nosso estudo, foi atribuído à neurotoxoplasmose cursando com hipertensão intracraniana ${ }^{20}$.

Outros achados não relacionados à infecção por T. gondii foram igualmente importantes, porque permitiram o diagnóstico de alterações fundoscópicas que comprometem a qualidade de vida do paciente. Nesse grupo, estiveram incluídos o aumento da escavação papilar, secundário a glaucoma crônico de ângulo aberto, e a degeneração retiniana periférica (lattice), presente em um paciente com miopia de alta dioptria. Ambos os pacientes foram submetidos a tratamento oftalmológico profilático, com a intenção de prevenir o descolamento de retina. 
Concluiu-se que pacientes com AIDS e neurotoxoplasmose podem apresentar alterações fundoscópicas características da toxoplasmose ocular, seja na forma ativa ou cicatricial, relacionadas ao HIV ou, ainda, a outras doenças oportunistas ou sistêmicas. podendo ser de grande auxílio no tratamento do paciente.

\section{CONFLITO DE INTERESSE}

Os autores declaram não haver nenhum tipo de conflito de interesse.

\section{REFERÊNCIAS}

1. Belfort R. The ophthalmologist and the global impact of the AIDS epidemic: LV Edward Jackson Memorial Lecture. Am J Ophthalmol 2000; 1:1-8.

2. Lewallen S, Courtright P. HIV and AIDS and the eye in developing countries. Arch Ophthalmol 1997; 115:1291-1295.

3. Cochereau-Massin I, LeHoang P, Lautier-Frau M, Zerdoun E, Zazoun L, Robinet $\mathrm{M}$, et al. Ocular toxoplasmosis in human immunodeficiency virus-infected patients. Am J Ophthalmol 1992;144:130-135.

4. Carmo EL, Almeida EF, Bichara CN, Póvoa MM. Pesquisa de anticorpos antiToxoplasma gondii em fluidos intra-oculares (humor vítreo e humor aquoso) de pacientes com toxoplasmose ocular, na cidade de Belém, PA. Rev Soc Bras Med Trop 2005; 38:77-79.

5. Vargas MA, Rodrigues MLV, FigueiredoJFC, Souza NV. Condições oftalmológicas de pacientes com síndrome da imunodeficiência com longo tempo de seguimento. Arq Bras Oftalmol 2007; 70:85-89.

6. Palella Jr FJ, Delaney KM, Moorman AC, Loveless MO, Fuhrer J, Satten GA, et al. Declining morbidity and mortality among patients with advanced human immunodeficiency virus infection. HIV Outpatient Study Investigators. N Engl J Med 1998; 338:853-860.

7. Glasner PD, Silveira C, Kruszon-Moran D, Martins MC, Burnier Júnior M, Silveira $S$, Camargo ME. et al. An unusually high prevalence of ocular toxoplasmosis in Southern Brazil. Am J Ophthalmol 1992; 114:136-144

8. Humphry RC, Weber JN, Marsh RJ. Ophthalmic findings in a group of ambulatory patients infected by human immunodeficiency virus (HIV): a prospective study. Br J Ophthalmol 1987; 71:565-569.

9. Weiner A, BenEzra D, Clinical patterns and associated conditions in chronic uveitis. Am J Ophthalmol 1991;112:151-158.

10. Auzemery A, Queguiner P, Georges AJ, Di Costanzo B, Georges-Courbot MC, Vohito MD. Ophthalmologic manifestations of acquired immunodeficiency syndrome (AIDS) in central Africa. Med Trop 1990; 50:441-443.

11. Gerente VM, Spada FR, Santos EM, Rossi EE, Coelho AI. Manifestações oculares de pacientes HIV positivos atendidos no Serviço de Oftalmologia do Hospital Regional de São José - SC. Arq Catarinenses Med 2004; 33:27-32.

12. Sampaio VL, Lucena AR, Cantanhede TMI, Sampaio TL, Dias S. Achados oculares em pacientes HIV positivos em Pernambuco, Brasil. Fac Med Univ Fed Pernamb Recife 2000; 45:126-130.

13. Centers for Disease Control. 1993 revised classification system for HIV infection and expanded surveillance case definition for AIDS among adolescents and adults. JAMA 1993; 10:729-730.

14. Arruda RF, Muccioli C, Belfort Jr R. Achados oftalmológicos em infectados pelo HIV na era pós-HAART e comparação com série de pacientes avaliados no período pré-HAART. Rev Assoc Med Bras 2004; 50:148-152.

15. Matos KFT, Santos MCM, Muccioli C. Manifestações oculares do paciente infectado pelo HIV atendido no Departamento de Oftalmologia da Universidade Federal de São Paulo. Rev Ass Med Brasil 1999; 45:323-326.

16. Muccioli C, Belfort Jr R, Lottenberg C, Lima J, Santos P, Kim M, et al. Achados oftalmológicos em AIDS: avaliação de 445 casos atendidos em um ano. Rev Ass Med Brasil 1994; 40:155-158.

17. Zajdenweber M, Muccioli C, Belfort Jr R. Ocular involvement in AIDS patients with central nervous system toxoplasmosis - before and after HAART. Arq Bras Oftalmol 2005; 68:773-775.
18. Goldberg DE, Smithen LM, Angelilli A, Freeman WR. HIV-associated retinopathy in the HAART era. Retina 2005; 25:633-649.

19. Vrabec TR. Posterior segment manifestations of HIV/AIDS. Surv Ophthalmol 2004; 49:131-157.

20. Oréfice F. Uveíte clínica e cirúrgica. Texto \& Atlas. Rio de Janeiro: Cultura Médica. 2000; v. 1-2. 